\title{
Measurements of Acoustic Radiation of Tonewood Spruce at Different Clamping Loads
}

\author{
J. GÖKEn ${ }^{a, *}$, N. SABA ${ }^{b}$ And K. SteINHOFF ${ }^{c}$ \\ ${ }^{a}$ University of Applied Sciences Emden/Leer, Faculty of Maritime Sciences, \\ Bergmannstraße 36, 26789 Leer, Germany \\ ${ }^{b}$ University of Balamand, Faculty of Engineering, Department of Mechanical Engineering, \\ Koura campus, 100 p.o. Box Tripoli, Lebanon \\ ${ }^{c}$ University of Kassel, Chair of Metal Forming Technology, \\ Kurt-Wolters-Straße 3, 34125 Kassel, Germany
}

Received: 03.09.2021 \& Accepted: 13.12.2021

Doi: $10.12693 /$ APhysPolA.141.54

*e-mail: juergen.goeken@hs-emden-leer.de

\begin{abstract}
The usage of wood as an environmentally friendly material combines optimized room aesthetics with mechanical and/or acoustic functionality. Cellular materials like wood play an important role in reducing vibration and noise. It is considered to have low damping properties due to its molecular structure and its relatively high modulus of elasticity. This property is well known to musical instrument makers and is accordingly taken into consideration in the manufacture of such instruments. Site-specific, targeted wooden cladding can help to dampen vibrations. Against the background of noise and vibration control, wooden plates in the form of connected bodies are often used, e.g. to build an enclosure. The vibration behaviour of the resonance body of a wooden musical instrument is difficult to determine. In this context, basic acoustic investigations into the radiation efficiency of wooden panels become mandatory. The solutions to the equation of motion of the vibrating plate depend, among others, on the geometry of the plate and the conditions of its clamping. A fact that makes it very challenging to predict is e.g. eccentric load application that causes much more natural vibrations. In this paper, a plate made of the tonewood spruce was mechanically excited in the centre on the back. The acoustic intensity of the panel on the other side was then measured as a spatial function using an acoustic sensor. Since the clamping conditions influence the vibration characteristics, the measurements were conducted under different clamping loads. A relatively stable mode pattern emerged that was more pronounced at the highest clamping load.
\end{abstract}

topics: tonewood, radiation behaviour, acoustic measurement

\section{Introduction}

In many applications, especially in the transport sector, components or machines are often exposed to cyclic loads during service time. Investigations into material fatigue are therefore of considerable technological interest, especially especially as it allows targeted material substitution to be carried out. Loading frequencies during fatigue tests are performed near the resonance frequency of the system $[1,2]$. While resonance vibrations in dynamically stressed components are rather undesirable, in the construction of musical instruments (e.g. string instruments) the development of tones would not be possible without them. In order to optimise the sound of musical instruments or to make a quality classification, modal analysis is necessary. Modal analysis is often carried out using the roving hammer method or laser Doppler vibrometer [3]. Moreover, holographic interferometry is a useful technique for studying the vibrational modes of both separate vibrating elements and complete instruments [4]. In a current work of Rucz et al. [5], a coupled vibroacoustic model of the sound generation by mallet percussion instruments was discussed. A mechanical model based on the Navier-Cauchy equations for linear elasticity was combined there with an acoustical model describing the propagation of acoustical waves. The proposed approach relies on the 3D FEM.

The modal analysis of oscillating systems is also necessary for offshore activities. Here, for example, the resonance behaviour of electrical submersible pumps is investigated. The system is excited by an impact hammer, whereas the corresponding response signals were acquired by accelerometers [6]. A similar procedure can be performed on the violins to determine their modal response [7].

Civil engineering is another area where modal analysis is playing an increasing role. The impact of vibrations transmitted through the ground on buildings is investigated using "Ansys" engineering 
simulation [8]. In this context, modal analysis is also used to understand the dynamic properties of buildings under seismic excitation [9]. In order to obtain modal parameters of bridges, the digital image correlation (DIC) method is used [10]. The DIC method is an optical deformation measurement technique (noncontact detection method) that can be used to measure the three-dimensional, wholefield deformation of a body. In addition to that, the dynamic response of typical rotating structures, such as helicopter rotor blades and wind turbine blades, is a challenging area of research in structural analysis and uses the DIC method as well [11]. Contactless acoustic measurements are the most beneficial when used to reduce the amplitude of dynamic instabilities or resonances in large structures like plates or enclosures. In plate-like structures, the thickness of which is small relative to the wavelength (Kirchhoff plate theory), bending waves are the most important wave type for the emission of secondary airborne sound. The transverse deflection of the surface of the vibrating object leads to the radiation of the sound into the surrounding air. The sound field created by the vibration of finite objects is affected by the shape and dimensions of the surface. There is a distribution of vibrations on surface, which is dependent on the vibration amplitude, its phase and frequency. Moreover, the velocity of propagation of the bending waves in plates depends on the excitation frequency (dispersion). The sound radiation of the vibrating plate is made up of the superposition of the modal radiation of all elementary oscillation forms involved [12].

Boundary conditions have a significant influence on the sound radiated from rectangular plates as found by Qiao et al. [13]. It is expected that with varying boundary conditions, the number of frequency-dependent peaks of the sound power level radiated from the plates will vary. Thus, in order to control the sound, the natural frequencies of vibrations of rectangular plates at different boundary conditions have to be determined.

The work of Leissa [14] deals intensively with the emergence of oscillation modes of plates under different clamping conditions and has for years been considered the standard literature. Maidanik [15] predicted the sound radiation efficiency for finite rectangular plates, which was later improved by Leppington et al. [16] using energy transfer analysis. Zou and Crocker [17] emphasise the importance of boundary conditions, since when the amplitudes of some of the modes are reduced, the amplitudes of some of other modes can be increased at the same time.

The construction of musical instruments uses wooden panels to build resonance bodies. In this particular industrial sector, knowledge of the vibration behaviour of wooden panels is essential as it is critical for the sound of the musical instrument $[18,19]$. Thus, an important part of the structural dynamics and, especially of the vibroacoustics,

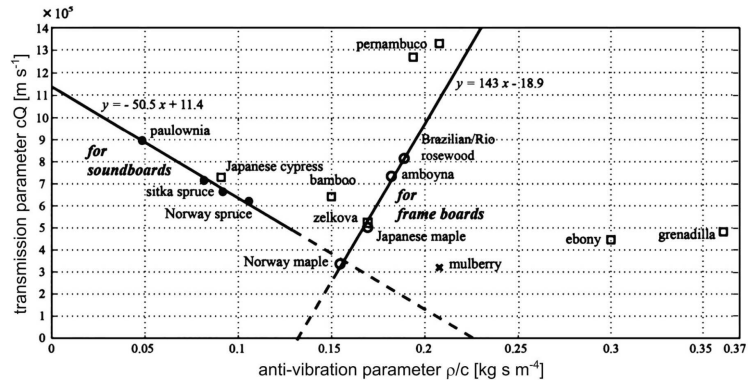

Fig. 1. Classification diagram of traditional woods for string instruments and for other instruments. -: tone woods for the soundboard; ○: frame woods; $\times$ : traditional wood for the Satsuma biwa; $\square$ : traditional woods for other instruments; taken from Ref. [22]; the font of the axis label has been adjusted.

is the calculation of the natural modes of vibration of a plate. However, there is a lack of information on how strongly the clamping conditions influence the vibration behaviour of wooden instruments and, thus ultimately, their sound. Tuning the sound remains a major physical-technical challenge in the field of musical instruments.

The modulus of elasticity together with the density determines most of the acoustical properties of a cellular material like wood, as shown by Wegst [20]. Classification diagram of traditional woods for the construction of musical instruments based on the anti-vibration parameter (density $\rho /$ sound speed $c$ ) and the transmission parameter $c Q$ ( $Q$ is the quality factor, i.e., the reciprocal of the loss factor, which is determined by the internal friction (damping) of wood [21]) was proposed by Yoshikawa and Waltham [22] (see Fig. 1).

A classification by Golovin [23] states that the value of low damping material (in terms of tangent $\tan (\delta))$ is below 0.1 , whereas that value for high damping is considered to be above 1. Therefore, wood can generally be considered a low damping material. It must be pointed out that the damping behaviour depends on various parameters (e.g. fibre direction, moisture content, age) [24]. Especially the moisture content was found to have a strong influence on the damping [25]. This makes it difficult to predict whether the wooden material has high or low damping properties.

One of the most important tonewoods is spruce wood due to its good resonance properties. As can be seen in Fig. 1, spruce wood is especially used for soundboards, which are the primary source of sound [26]. The wood is the preferred construction material for upright and grand pianos, bowed instruments (violin, viola, cello, double bass) and plucked instruments (guitar, harp, zither).

In this work, it was investigated to what extent the clamping load has an influence on the radiation of wooden plates which is characterized by the sound pressure level and its distribution. 
TABLE I

Specifications of the used woods from different references. Note: ${ }^{*}$ technical value at $\sim 12 \%$ moisture content

\begin{tabular}{l|l|c|c|c}
\hline \hline \multicolumn{1}{c|}{ Samples } & \multicolumn{1}{c|}{ Botanical name } & $\begin{array}{c}\text { Specific density* } \\
\text { (air-dry) }\left[\mathrm{g} / \mathrm{cm}^{3}\right]\end{array}$ & $\begin{array}{l}\text { Modulus of elasticity* } \\
\text { (bending) }\left[\mathrm{N} / \mathrm{mm}^{2}\right]\end{array}$ & $\begin{array}{c}\text { Years } \\
\text { of growth }\end{array}$ \\
\hline Spruce wood [30] & Picea abies & 0.40 & 10800 & $\sim 40$ \\
American maple wood [30] & Acer saccharum & 0.57 & 11200 & $\sim 80$ \\
European maple wood [31] & Acer pseudoplatanus L. & 0.63 & 10900 & $\sim 80$ \\
American cherry wood [30] & Prunus serotine & 0.50 & 10300 & $\sim 80$ \\
European cherry wood [32] & Prunus avium & 0.54 & 10600 & $\sim 80$
\end{tabular}

\section{Experimental details}

The acoustic measurements were carried out on five wooden plates (length: $505 \pm 1 \mathrm{~mm}$, width: $148 \pm 1 \mathrm{~mm}$, thickness: $1.5 \pm 0.1 \mathrm{~mm})$. A wooden panel made of spruce, American and European maple wood as well as American and European cherry wood were available. The data of the measurements to be performed may only be considered as possible results because of the small number of samples. In the work of Downes and Drew [27] it is pointed out that different mechanical properties are to be expected even in wood samples from neighbouring areas of the tree. This is to illustrate that the results on the mechanical properties of samples of the same origin can differ considerably. Therefore, statistical statements on a biological material like wood are generally difficult.

Maple wood and cherry wood were applied in order to investigate whether the radiation behaviour of woods is similar or specific. Since spruce wood, which functions as tonewood, is offered in particular with varying quality (e.g. as construction timber), it should be emphasised at this point that the spruce wood used in this work came from altitudes of 1200 meters up to the vegetation limit. It is usually characterized by narrow annual rings, resulting in higher stiffness, which is necessary for its use as a tonewood. However, the focus on the narrowness of the annual rings is only one criterion among several others for classifying a particular wood as a tonewood [28]. Spruce wood serves, among other things, as a material for the top plate of guitars.

The wood samples were taken from the same cutting direction and exposed to room temperature. Moisture content of wood depends significantly on temperature and relative air humidity [29]. At room temperature, the moisture content of wood is expected to be around $10 \%$. It is assumed that this parameter is to be considered equally weighted for all samples. The specimens were not aged (no degenerated wooden cells) so that a comparison of the acoustic properties of these woods is possible. The individual specification of each wood is listed in Table I [30-32].

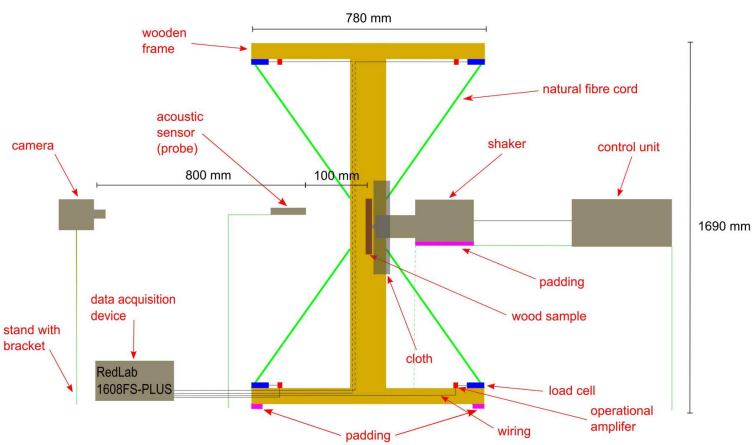

Fig. 2. Side view of the experimental setup.

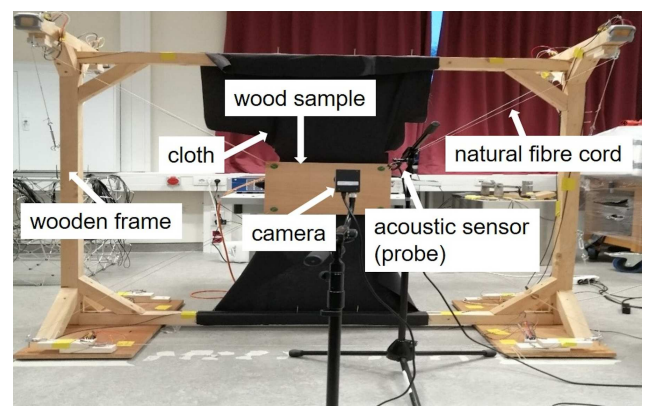

Fig. 3. Front view of the experimental setup.

Each wooden plate was clamped in a wooden frame with the help of natural fibre cords (see Fig. 2). To prevent the structure from absorbing external vibrations or emitting them to the outside, the wooden frame was mounted on rubber feet which act as padding. Figure 3 demonstrates the real experimental setup of the schematic drawing shown in Fig. 2.

A hole was cut in each corner of the single wooden sample to realize the suspension. The holes of the rectangular sample were fitted with plastic sleeves produced by a 3D printer. This enabled the cords to be guided better and minimized their dry friction (external damping) in the holes.

In order to ensure uniform tensile stress at all fixing points, each of eight strings was fitted with a load cell (model CZL635; Tinkerforge $\mathrm{GmbH}^{\mathrm{TM}}$, 


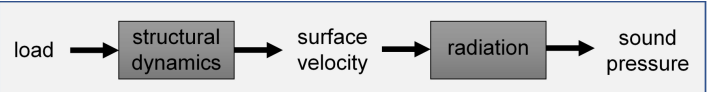

Fig. 4. Signal flow diagram of the indirect sound radiation; translated and adopted from [12].

Schloß Holte-Stukenbrock, Germany) that output electrical voltage. The output voltage had to be amplified using an operational amplifier. This electrical voltage was then converted into a unit of mass. The specification of the mass was chosen because the own calibration of the load cells was also carried out by means of attached masses. As a result of the correlation between mass and indicated output voltage of the load cells, a load representing a specific mass (denoted as "clamping load") could be set by changing the tensile stress in the fibre cords.

From the operational amplifier, the signal was passed on to a data acquisition device. At its 8 analogue connections, the analogue data were digitally converted (16 bit A/D converter, model RedLab 1608FS-PLUS; Meilhaus Electronic $\mathrm{GmbH}^{\mathrm{TM}}$, Alling, Germany).

On one side of the sample, a vibration exciter (electrodynamic shaker, model TV 50018; TIRA $\mathrm{GMBH}^{\mathrm{TM}}$, Schalkau, Germany) was placed in the centre of the plate. The shaker was connected to a control unit with which the excitation frequency can be regulated. The excitation frequencies were $82.4 \mathrm{~Hz}, 146.8 \mathrm{~Hz}, 220 \mathrm{~Hz}, 440 \mathrm{~Hz}$ and $659.3 \mathrm{~Hz}$. These frequencies respectively represent the notes E, d, a, a', e" of a commercially available acoustic guitar which was taken as reference for classical stringed instruments.

The shaker exerted a nominal force of $18 \mathrm{~N}$ on the wood. A cloth was stretched between the wood sample and the shaker, which should prevent additional excitation of the wood by the sound radiation of the exciter.

The vibration excitation on the back of the wood leads to structure-borne sound, which causes a radiation behaviour on the front of the wooden panel. The resulting sound pressure distribution was determined by measuring the local sound pressure level using a sound source localization system (acoustic sensor; Microflown ${ }^{T M}$, Arnhem, The Netherlands). Using this probe the radiation efficiency of a stainless-steel plate could be investigated [33]. In a former work the vibrating pattern were recorded and compared with Chladni figures of a vibrating plate [34].

The basic measuring process is illustrated in Fig. 4. All results shown here were given in $\mathrm{dB}(\mathrm{A})$ and visualised by colour-coding. Positions with the highest sound pressure level were colour-coded in red, positions with the lowest sound pressure level were colour-coded in blue.

Additionally, a digital camera was used, the picture of which was correlated with the positions and

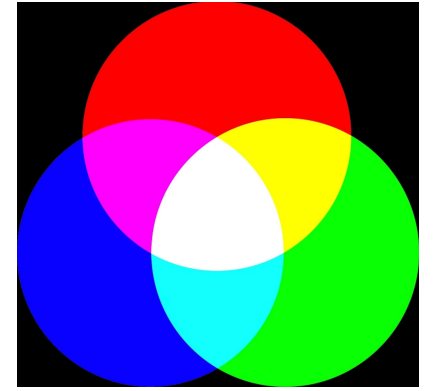

Fig. 5. Colour-mixing of RGB values.

the values of the measured sound pressure level. As can be seen in Fig. 2, the probe was placed at a distance of $100 \mathrm{~mm}$ from the sample. The camera was located at a distance of $800 \mathrm{~mm}$ from the front of the probe.

The wood samples were divided into approximately equal-sized imaginary sections, each containing one measuring point. This subdivision was chosen so that there was an even distribution from the excitation point to the clamping points or the edges of the sample. Measurements were taken at 14 points on the wooden panels to be compared. The probe was pointed at the respective measuring point while the shaker was exciting the wood. Maintaining same distance between the probe and the sample during each measurement was ensured. For the measurement of the locally occurring sound pressure level change, the acoustic measuring sensor was guided parallel along the panel surface.

The representation of colour-coded acoustic images is based on the superposition of the primary colours "red" (R), "green" (G) and "blue" (B). Each colour includes an intensity value from 0 to 255 . Considering Fig. 5, it becomes clear to which colours the individual pairs of primary colours add up. Points in the image that lie in the average of all three circles are displayed in white.

A region of interest was selected from each wooden plate (equal area size) and evaluated based on the proportions of RGB values (see Fig. 6). For this purpose, the open source image processing program "ImageJ" [35] was used, which allows a useradapted RGB evaluation.

Detailed exposure of the region of interest is shown in Fig. 7. To obtain a representative distribution of RGB values, a "zigzag line" was drawn from one end of the section to the other. The total number of data points collected for the RGB analysis was approximately 2850. It was found that this amount of data is sufficient to distinguish areas of high vibration intensity (colour-coded in red) from areas of low vibration intensity (colour-coded in blue).

In order to determine the influence of the clamping load on the sound pressure level and its distribution at constant excitation frequency, the clamping values at the measuring cells were varied. 


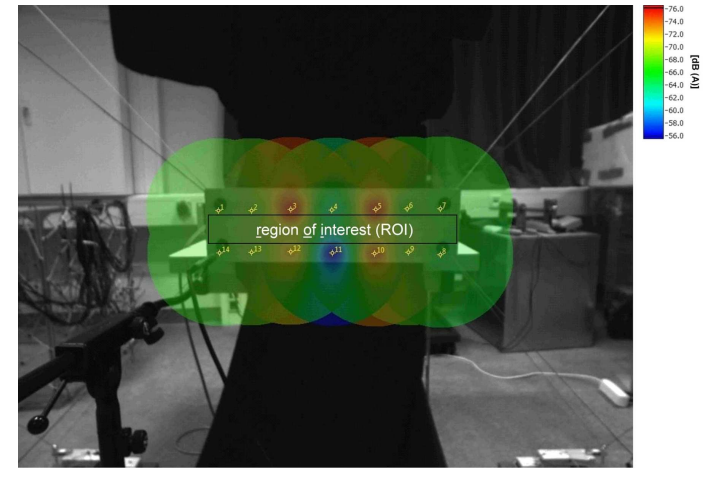

Fig. 6. Colour-coded sound pressure measurement demonstrating the region of interest for subsequent evaluation.

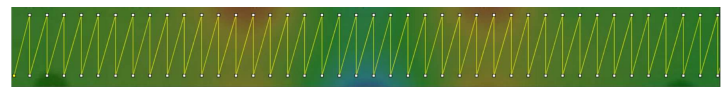

Fig. 7. Drawn zigzag line for investigation of RGB-values in the region of interest.

The following clamping values (by changing the tensile stress in the fibre cords) were adopted for investigation $200 \mathrm{~g}, 400 \mathrm{~g}, 500 \mathrm{~g}, 600 \mathrm{~g}, 800 \mathrm{~g}$.

\section{Results and discussion}

\subsection{Results}

In Fig. 8, the intensity of each RGB colour of spruce wood is plotted versus the distance from the left clamping point to the right clamping point of the panel. To ensure the reproducibility of the results, two measurements were performed. The letter "C" represents the centre of the wooden panel. The centre is also the place where the shaker introduces external vibration energy. The measurement was carried out under an excitation frequency of $440 \mathrm{~Hz}$ (concert pitch a) and a clamping load of $600 \mathrm{~g}$.

It is noticeable that the measurement has a high proportion of green over the entire measuring range. It seems to be a kind of fundamental radiation because it occurs relatively evenly over the region of interest. The blue component is increased, especially in the centre. The area with the highest sound pressure level is arranged symmetrically around the centre. In general, it can be determined that the vibration energy (red colour) decreases in the direction of the edges of the clamped plate. At the location of the vibration excitation, the blue colour predominates, which means that the vibration energy in this area is low. The spruce plate is ultimately characterized by two dominant vibration modes representing resonant modes of vibration.

In contradictory to the expectation that the blue colour will rise towards the edges as a direct result of the clamping conditions, the results showed

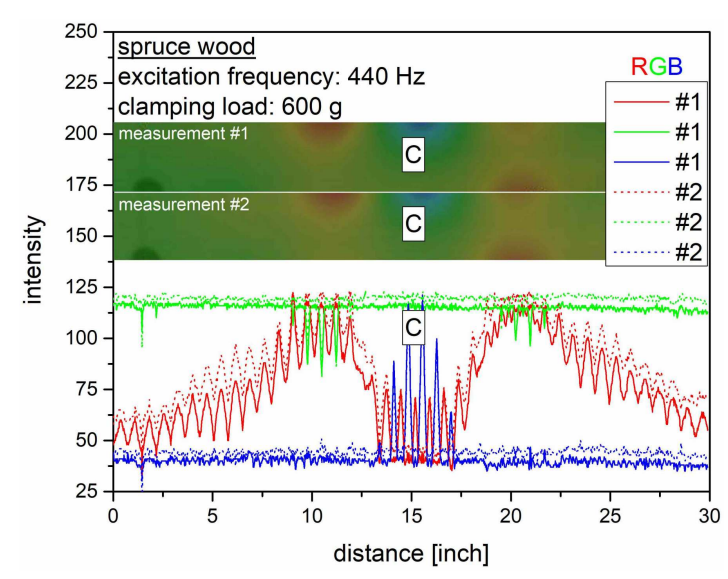

Fig. 8. Colour-coded sound pressure distribution on a spruce plate that were excited to vibrate.

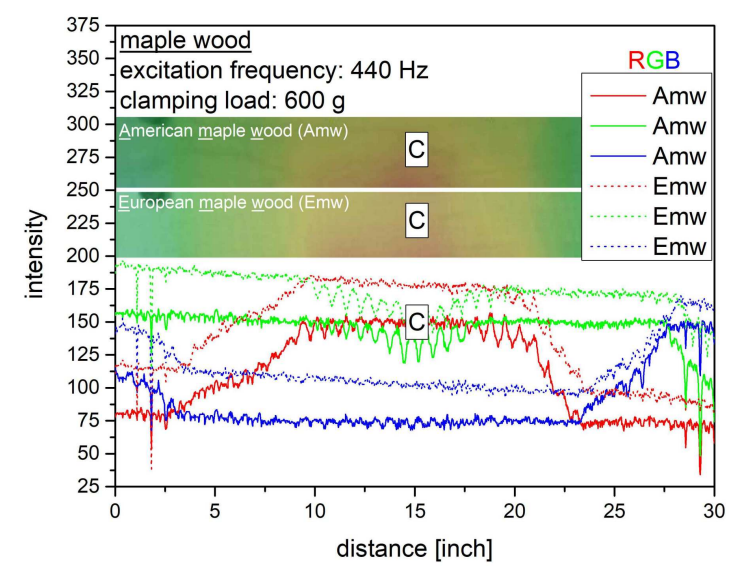

Fig. 9. Colour-coded sound pressure distribution on different maple plates that were excited to vibrate (American maple wood (Amw), European maple wood (Emw)).

that the spruce wood apparently has the property of distributing vibrational energy relatively evenly over the radiating surface.

Another tonewood (American and European maple, see Figs. 1 and 9) was used for comparison. Despite different places of origin, both types of woods show a similar pattern of sound pressure level distribution, which is made clear by the colour.

In contrast to spruce wood, a maximum sound pressure level can be seen in the area in which the excitation energy is introduced (red colour). There is obviously a different sound pressure distribution compared to spruce. In addition, RGB values are overall larger (higher intensity), which indicates that less excitation energy is destroyed in the case of maple wood. Consequently, the energy dissipation is lower, i.e., maple wood possesses less damping capacity than spruce, which can be attributed to the higher modulus of elasticity of this wood [24] compared to spruce (see Table I). 


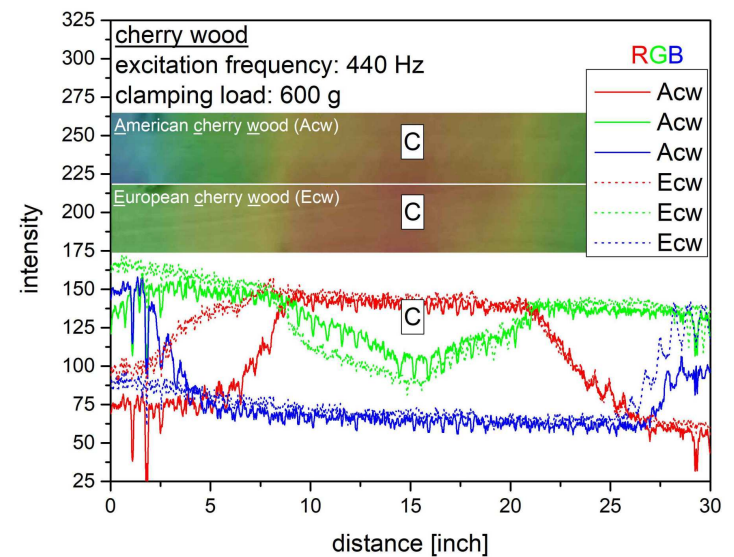

Fig. 10. Colour-coded sound pressure distribution on different cherry plates that were excited to vibrate (American cherry wood (Acw), European cherry wood (Ecw)).

It can be seen that the intensity of the red and green colour is reduced in the direction of the clamping locations, while - on the other hand - the intensity of the blue colour increases. This is a sign of the dominance of clamping force, which seems to predominate for this wood because of its previously mentioned lower damping capacity. Although the plate geometry was approximately the same, both American and European maple wood vibrate differently to spruce wood. Apparently, only one broad resonant mode of vibration prevails that carries almost all of the vibrational energy.

The results of the acoustic radiation measurements of American and European cherry wood are shown in Fig. 10.

In general, cherry wood is not considered to be a tonewood, but it has comparable radiation behaviour as maple. However, the intensity seems to be a little less. The used American and European cherry woods have very similar radiation behaviour among themselves. It becomes clear that the fundamental radiation (green colour) in the area of the energy input is reduced. This reduction is higher than in maple. The radiation characteristic in the centre is essentially dominated by the red colour. Here, a fading of the red and green colour can be observed in the direction of the clamping point as well. This means that the blue colour increases in this area, indicating a significant reduction in radiation. As in the case of the maple, the radiation behaviour is essentially determined by one resonant mode of vibration. According to [36], the material damping (in terms of loss tangent $\tan (\delta)$ ) of cherry wood is about 0.01 , and of maple wood about 0.008 [37]. For spruce wood, the value of $\tan (\delta)$ is nearly 0.03 [38]. Former measurements have shown how strongly this value depends on the moisture content and age of the wood [24]. Therefore, the damping values given can only be considered as guide values. The modulus of elasticity of cherry wood is similar to that of

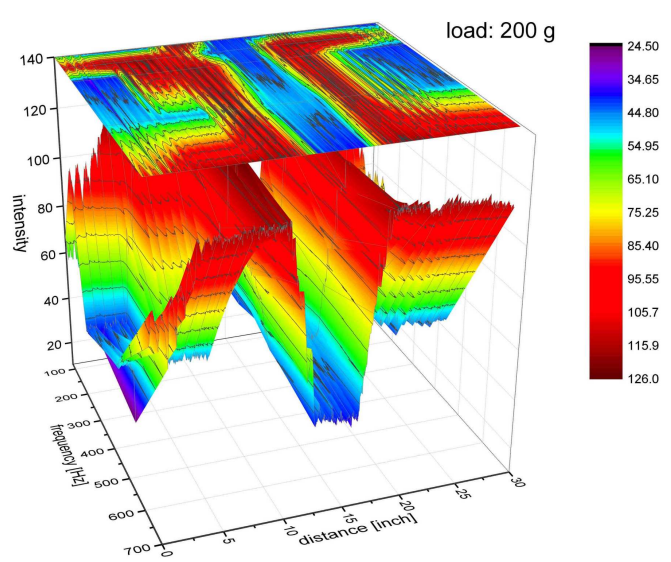

Fig. 11. Dependence of the colour intensity on the excitation frequency and the location of the acoustic sound radiation of spruce wood; clamping load: $200 \mathrm{~g}$.

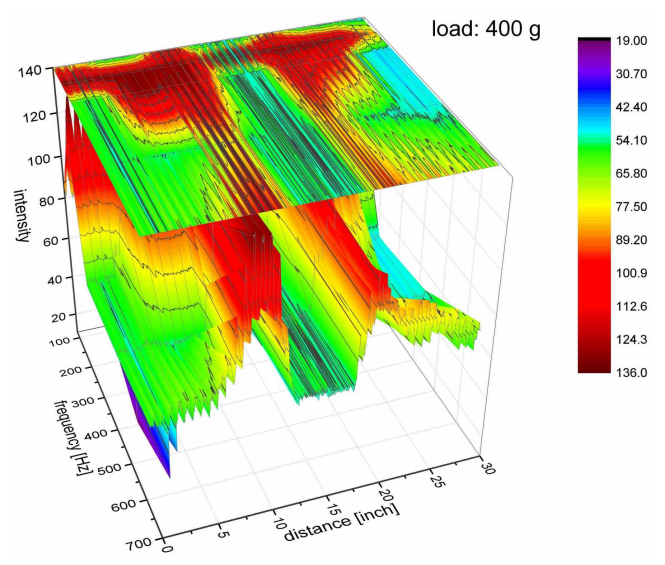

Fig. 12. Dependence of the colour intensity on the excitation frequency and the location of the acoustic sound radiation of spruce wood; clamping load: $400 \mathrm{~g}$.

spruce wood (Table I), which can explain the overall lower intensity of the colours due to the higher material damping of cherry wood compared to maple wood. Since spruce wood and maple wood are established tonewoods (see Fig. 1), cherry wood has the potential to function as a tonewood due to its comparable acoustic properties.

In Figs. 11-15 the RGB colour intensities as a function of the excitation frequency and the location of the sound radiation of spruce wood are shown. Measurements differ with regard to the clamping load (200 g, $400 \mathrm{~g}, 500 \mathrm{~g}, 600 \mathrm{~g}$, $800 \mathrm{~g})$. The three-dimensional plot was projected onto a surface to better estimate the intensity distribution.

At a load of $200 \mathrm{~g}$ (see Fig. 11), the curve of the acoustic radiation intensity is similar to that shown in Fig. 8. There are two oscillation maxima. The area of excitation is indicated by a blue colour which corresponds to a vibration node in the centre 


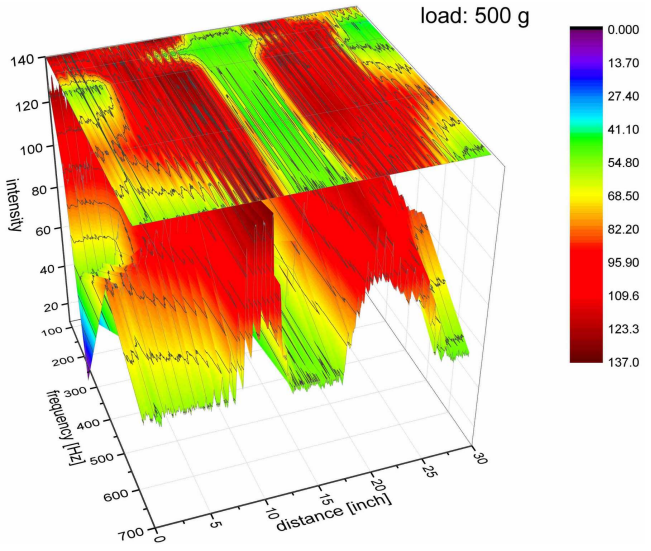

Fig. 13. Dependence of the colour intensity on the excitation frequency and the location of the acoustic sound radiation of spruce wood; clamping load: $500 \mathrm{~g}$.

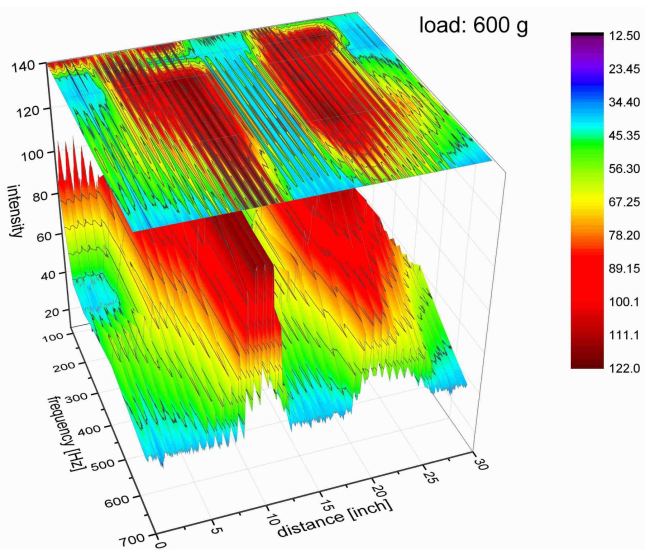

Fig. 14. Dependence of the colour intensity on the excitation frequency and the location of the acoustic sound radiation of spruce wood; clamping load: $600 \mathrm{~g}$.

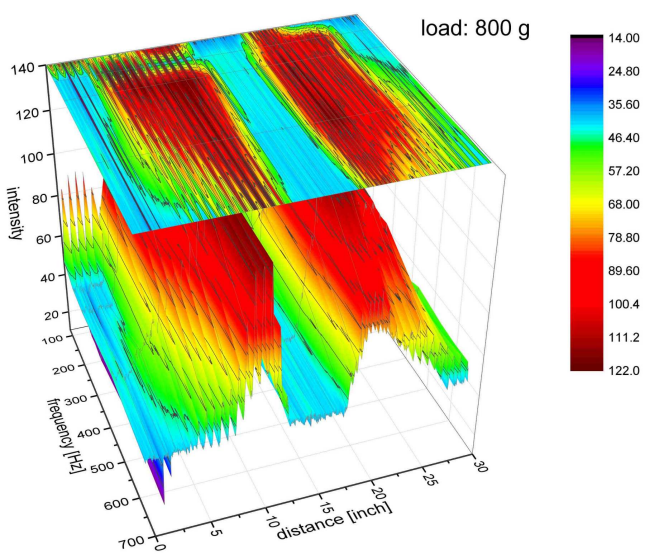

Fig. 15. Dependence of the colour intensity on the excitation frequency and the location of the acoustic sound radiation of spruce wood; clamping load: $800 \mathrm{~g}$. of the plate. It is evident that the oscillation energy continues towards the corners where the plate is suspended.

The area between the corners of the short side appears to be vibration-free. The scale shows that the difference between the lowest and the highest colour intensity amounts to approximately 101 scale units. In summary, a mixed radiation pattern occurs.

A significant difference in results can be seen in the case of $400 \mathrm{~g}$ load (see Fig. 12). The oscillation maxima are less pronounced and the central area of the sample seems to vibrate more. Moreover, the difference between the lowest and highest colour intensity is about 117 scale units. The vibrational energy in the direction of the suspension is not equally distributed.

However, a very strong expression of the oscillation maxima can be observed at a load of $500 \mathrm{~g}$ (see Fig. 13). The area in which the energy is introduced emits vibrational energy also in this loading condition. Less vibration radiation can be measured at the locations of suspension. In summary, it can be mentioned that the sample shows a more balanced vibration pattern compared to $400 \mathrm{~g}$ load. The difference in intensity values increases to 137 scale units. A very strong expression of the oscillation maxima can, however, be observed at a load of $500 \mathrm{~g}$ (see Fig. 13). The area in which the energy is introduced emits vibrational energy also in this loading condition. Less vibration radiation can be measured at the locations of suspension. In summary, it can be mentioned that the sample shows a more balanced vibration pattern compared to $400 \mathrm{~g}$ load. The difference in intensity values increases to 137 scale units.

With a load of $600 \mathrm{~g}$, the vibrational energy is significantly reduced to the clamping points (see Fig. 14). One recognizes, however, clear maxima of radiation around the centre of the wooden plate. These reach almost to the edge of the plate. At the point of excitation of the vibration, there hardly takes place any radiation. The measured difference between the highest and the lowest intensity is about 110 scale units.

In the case of the highest clamping load of $800 \mathrm{~g}$ (see Fig. 15), there is an even more pronounced differentiation of the radiation pattern. Now, the locations of the highest radiation are almost concentrated exclusively in the area around the centre of the plate. The centre and clamping points barely oscillate. The difference between the minimum and maximum intensity is about 108 scale units. The plate edges radiate insignificant vibrational energy.

\subsection{Discussion}

In the following, a rectangular plate with the edge lengths $a$ and $b$ is to be considered that is simply supported in an infinite rigid baffle. Here, the $z$-coordinate represents the direction in which the 

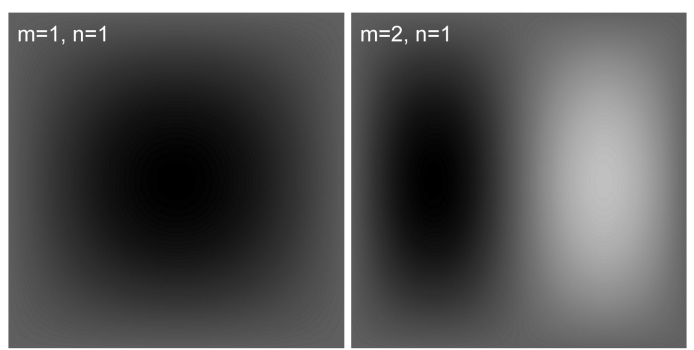

Fig. 16. Expected natural modal shape evolution according to (1) for $m=1, n=1$ (left) and $m=2$, $n=1$ (right).

plate can be bowed out. For a stationary plate surface, $z=0$ is valid. The natural modal shape $W$ of the plate is given by [39]

$$
W_{m, n}(x, y)=\frac{2}{\sqrt{a b}} \sin \left(\frac{m \pi}{a} x\right) \sin \left(\frac{n \pi}{b} y\right)
$$

with $m, n=1,2, \ldots$ (mode numbers); $x$ and $y$ represent coordinates whereas $0 \leq x \leq a$ and $0 \leq y \leq b$. The numerical values $(m-1)$ or $(n-1)$ correspond to the number of oscillation nodes of these modes in $y$ - or $x$-direction. In Fig. 16 the calculated natural modal shapes $W_{1,1}$ and $W_{2,1}$ of the plate with $a=505 \mathrm{~mm}$ and $b=148 \mathrm{~mm}$ are shown.

According to the calculated natural modal shape for maple and cherry wood, only the main mode $\left(W_{1,1}\right)$ is activated (see Fig. 9 and 10). Vibration behaviour of spruce wood seems to be dominated by 2 modes ( $W_{2,1}$, see Fig. 8). This may be explained by the lower specific density of spruce wood which is approximately 1.5 times lower than that of maple and cherry wood (see Table I). In contrast to spruce wood, the share of the blue and green colour in the case of maple and cherry wood is significant. Since the damping of spruce wood is higher, its energy distribution in the material is reduced.

With $\omega$ being the angular frequency and $\omega_{m, n}$ being the modal natural angular frequency of the plate, it can be written

$$
\omega_{m, n}=\sqrt{\frac{D}{m_{s}}}\left[\left(\frac{m \pi}{a}\right)^{2}+\left(\frac{n \pi}{b}\right)^{2}\right],
$$

where

$$
D=\frac{1}{12} \frac{E h^{3}}{\left(1-\nu^{2}\right)}
$$

is the bending stiffness of the plate with thickness $h$ and depends on the modulus of elasticity $E$ and the Poisson's ratio $\nu$ of the material (here: $\nu \approx 0.3$ ), while

$$
m_{s}=\rho h,
$$

is the surface density which can be calculated from the specific density $\rho$ and the plate's thickness. Using (2), the calculated natural angular frequency for $m=1$ and $n=1$ is $\omega_{1,1} \approx 922 \mathrm{~s}^{-1}\left(f_{1,1} \approx 147 \mathrm{~Hz}\right.$, $f$ is a frequency). For $m=2$ and $n=1$, one has $\omega_{2,1} \approx 1141 \mathrm{~s}^{-1}\left(f_{2,1} \approx 182 \mathrm{~Hz}\right)$.
Coincidence can be understood by realizing that the velocity of bending waves in a panel is a function of frequency. The coincidence frequency is of substantial importance for sound radiation since the degree of radiation depends on whether the frequency under consideration is below or above the coincidence frequency. The minimum value of the matching frequency occurs at grazing incidence and is called the critical frequency $f_{c}$. It is expressed as [40]

$$
f_{c}=\frac{\nu_{0}^{2}}{2 \pi} \sqrt{\frac{m_{s}}{D}}=\frac{\nu_{0}^{2}}{2 \pi h} \sqrt{\frac{12 \rho\left(1-\nu^{2}\right)}{E}}
$$

whereas $\nu_{0}$ is the sound speed in the air. For the used spruce wood plate, $f_{c} \approx 9.9 \mathrm{kHz}$ was calculated. In case of low frequency ratio, i.e., when the excitation frequencies are much lower than the critical frequency $f_{c}$, the damping becomes more dominant, which additionally complicates the calculation of the radiation pattern. In this study it can be assumed that the influence of the material damping remains constant, i.e., no dominance of this property develops.

Just as the plate oscillations under operating excitation result from the superposition of fundamental modes, the sound radiation of the vibrating plate is also the superposition of the modal radiation of all elementary vibration modes involved [12]. This fact underlines the difficulty of predicting the mode structure in a real case. Therefore, the excitation frequency $(82.4 \mathrm{~Hz}, 146.8 \mathrm{~Hz}$, $220 \mathrm{~Hz}, 440 \mathrm{~Hz}, 659.3 \mathrm{~Hz}$ ) does not have to correspond directly to the oscillation frequency $\left(f_{1,1} \approx\right.$ $147 \mathrm{~Hz}, f_{2,1} \approx 182 \mathrm{~Hz}$ ). The central excitation produces a fixed vibration pattern in spruce wood. It can be observed that the mode pattern stabilizes with increasing load. Especially, for excitation frequencies which produces less dominant vibration modes, the material damping becomes more important for the stability of the radiation efficiency [41]. Close to and above the critical frequency $f_{c}$ the radiation efficiency is not considerably affected by the boundary condition [41].

In the present paper, the used frequencies were far below $f_{c}$ and the boundary conditions must not be ignored. When the load is low, variations in the radiation efficiency can be expected in the edge regions. According to the work of Putra and Thompson [40], the influence of individual modes in the edge region has to be taken into account. It was found by Gomperts [42, 43] that plates with greater edge-constraints do not always have a greater radiation efficiency than less edge-constrained ones [44]. The radiation intensities shown in Figs. 11-15 suggest that the main modes appear to be only weakly dependent on the boundary loads. This was also found by Zhang and Li [45] for lowerorder modes. However, they demonstrated that the boundary conditions can have a significant impact on the modal radiation efficiencies and the radiated sound powers. 


\section{Conclusion}

The acoustic measurements were performed on an oscillating spruce wood panel. The rectangular wooden panel used was acoustically investigated at selected frequencies that range between $\approx 80 \mathrm{~Hz}$ and $660 \mathrm{~Hz}$ under different clamping loads. In order to compare the radiation characteristics, the radiation efficiency of the spruce panel was compared with that of European and American maple wood. In addition, a comparison was made between European and American cherry wood. The radiations from the maples were very similar. This also applied to cherry wood. The radiation efficiency of spruce wood was lower than that of other type of woods. Moreover, spruce wood showed two modes of vibration, while maple and cherry wood showed only one fundamental mode.

The modulus of elasticity of cherry wood tonewood is roughly between the value of spruce and maple wood. Both woods are considered tonewood. Due to the similar radiation efficiency, cherry wood can also be classified as tonewood which is relatively unknown. This result is advantageous from the point of view of musical instruments production, since cherry wood is very common in Europe.

The vibration pattern of spruce wood was examined under various clamping loads. The study showed that the mode pattern was not strongly dependent on the boundary loads. Nevertheless, the higher the loading force, there was a tendency towards more concise emission efficiency. The sound characteristics can thus be changed significantly through the clamping conditions. Spruce seems to have the ability to develop a stable and thus predictable mode pattern. However, the real natural frequencies of the vibrating wooden panel can deviate significantly from analytical values, as the clamping conditions must be observed. These conditions can have a lasting effect on the superposition of mode vibrations.

Wood is a cellular material. When considering its radiation efficiency, damping of the material must be taken into account. The damping is particularly important when the oscillation frequency of the rectangular wooden panel is far below the coincidence frequency.

The data shown here represent preliminary results. Differentiation of the used woods on the basis of their vibroacoustic behaviour seems to be possible but should be investigated in more detail in further studies for statistical reasons.

\section{Acknowledgments}

Prof. Dr. Jürgen Göken gratefully acknowledges the financial support of the German Research Foundation (www.dfg.de; DFG-reference number: INST 21572/1-1 LAGG).
The authors would also like to express their gratitude to the undergraduate student Mr. Wandersee for his scientific assistance and the wood supplier Otte Furniere - Hölzer, Wiesmoor, Germany, for providing the wood samples used in this work.

\section{References}

[1] W. Xu, Y. Zhao, X. Chen, B. Zhong, H. Yu, Y. He, C. Tao, Metals 10, 1415 (2020).

[2] F. Himmelbauer, M. Tillmanns, G. Winter, F. Gruen, C. Kiesling, Int. J. Fatigue 146, 106146 (2021).

[3] T. Duerinck, M. Kersemans, E. Skrodzka, M. Leman, G. Verberkmoes, W. Van Paepegem, in: 18th Int. Conf. of Experimental Mechanics, Brussels, 2018.

[4] T.D. Rossing, D.S. Hampton, in: Proc. SPIE 1396, Applications of Optical Engineering: Proc. of OE/Midwest '90, 1991, p. 108.

[5] P. Rucz, M.A. Ulveczki, J. Angster, A. Miklós, J. Acoust. Soc. Am. 149, 3200 (2021).

[6] R.S. Minette, S.F. SilvaNeto, L.A. Vaz, U.A. Monteiro, Ocean Eng. 124, 168 (2016).

[7] M. Mihalcica, M.D Stanciu, V.G Gliga, M. Campean, F. Dinulică, S.M. Nastac, IOP Conf. Ser.: Mater. Sci. Eng. 1182, 012048 (2021).

[8] K.R. Czech, Appl. Sci. 10, 3128 (2020).

[9] F. Pioldi, E. Rizzi, Earthquake Engng. Struct. Dyn. 47, 257 (2018).

[10] G. Chen, Z. Wu, C. Gong, J. Zhang, X. Sun, Adv. Civ. Eng. Mater. 2021, 6694790, 1 (2021).

[11] S. Rizo-Patron, J. Sirohi, Exp. Mech. 57, 367 (2017).

[12] P. Zeller, Körperschall, in: Handbuch Fahrzeugakustik, Ed. P. Zeller, Vieweg\&Teubner, Wiesbaden (Germany) 2012, ch. 7 (in German).

[13] Y. Qiao, Q. Huang, L. Li, J. Low Freq. Noise V.A. 26 2007, 115 (2007).

[14] A.W. Leissa, Vibration of Plates, Scientific and Technical Information Division, Office of Technology Utilization, National Aeronautics and Space Administration, Washington D.C. 1969.

[15] G. Maidanik, J. Acoust. Soc. Am. 34, 809 (1962).

[16] F.G. Leppington, E.G. Broadbent, K.H. Heron, Proc. R. Soc. Lond. A $\mathbf{3 8 2}$, 245 (1982).

[17] D. Zou, M.J. Crocker, Arch. Acoust. 34, 25 (2009). 
[18] N. Demoli, I. Demoli, Opt. Eng. 44, 090502 (2005).

[19] M.J. Elejabarrieta, A. Ezcurra, C. Santamariia, J. Acoust. Soc. Am. 111, 2283 (2002).

[20] U.G.K. Wegst, Am. J. Bot. 93, 1439 (2006).

[21] S. Yoshikawa, J. Acoust. Soc. Am. 122, 568 (2007).

[22] S. Yoshikawa, C. Waltham, in: Proc. of the Int. Symposium on Musical Acoustics, Le Mans (France) 2014, p. 281.

[23] I.S. Golovin, Metall. Mater. Trans. A 25, 111 (1994).

[24] J. Göken, S. Fayed, H. Schäfer, J. Enzenauer, Acta Phys. Pol. A 133, 1241 (2018).

[25] C.-T. Yeh, B.J. Hartz, C.B. Brown, J. Sound Vib. 19, 411 (1971).

[26] P. Dumond, N. Baddour, in: Proc. of the Int. Symposium on Musical Acoustics, Le Mans (France) 2014, p. 287.

[27] G.M. Downes, D.M. Drew, South. For. J. For. Sci. 70, 155 (2008).

[28] R. Viala, V. Placet, S. Cogan, J. Cult. Herit. 42, 108 (2020).

[29] P. Dietsch, S. Franke, B. Franke, A. Gamper, S. Winter, J. Civil Struct. Health Monit. 5, 115 (2015).

[30] D.E. Kretschmann, in: Wood Handbook Wood as an Engineering Material, General Technical Report FPL-GTR-190, United States Department of Agriculture, Forest Service, Forest Products Laboratory, Madison (WI) 2010, ch. 5.
[31] W. Sonderegger, A. Martienssen, C. Nitsche, T. Ozyhar, M. Kaliske, P. Niemz, Eur. J. Wood Prod. 71, 91 (2013).

[32] The wood database, 2021.

[33] S.C. Campbell, M.Sc. Thesis, University of Kentucky, 2019.

[34] J. Göken, H. Arends, H. Brink, ARPN J. Eng. Appl. Sci. 9, 1983 (2014).

[35] ImageJ, 2021.

[36] I. Brémaud, Y. El Kaïm, D. Guibal, K. Minato, B. Thibaut, J. Gril, Ann. For. Sci. 69, 373 (2012).

[37] S.A. Ahmed, S. Adamopoulos, Appl. Acoust. 140, 92 (2018).

[38] E. Rohloff, Z. Phys. 117, 64 (1941).

[39] Y. Kou, B. Liu, J. Tian, J. Acoust. Soc. Am. 137, 1032 (2015).

[40] A. Putra, D.J. Thompson, Appl. Acoust. 71, 1113 (2010).

[41] J.P. Arenas, Lat. Am. Appl. Res. 39, 345 (2009).

[42] M.C. Gomperts, Acta Acust. united Ac. 30, 320 (1974).

[43] M.C. Gomperts, Acta Acust. united Ac. 37, 93 (1977).

[44] G. Xie, D.J. Thompson, C.J.C. Jones, J. Sound Vib. 280, 181 (2005).

[45] X. Zhang, W.L. Li, J. Sound Vib. 329, 5307 (2010). 